HNO 2009 • 57:633-634

DOI 10.1007/s00106-009-1921-4

Online publiziert: 3. Juli 2009

(c) Springer Medizin Verlag 2009

\author{
C. Klingmann · P.K. Plinkert \\ Universitäts-Hals-Nasen-Ohren-Klinik Heidelberg
}

\title{
Neue Entwicklungen der Kochleaimplantation
}

mal. Defekte des Connexin-26-Gens spielen die Hauptrolle bislang bekannter genetischer Ursachen.

Seit dem Beginn der ersten Kochleaimplantationen in den 1970er-Jahren wurden weltweit mehr als 150.000 Systeme eingesetzt. Wurden zu Beginn der CI-Ära vornehmlich postlingual ertaubte Erwachsene versorgt, hat sich über die letzten 2 Dekaden das Indikationsspektrum stetig erweitert. Die Frühimplantation bei ertaubten Kindern ist heute in CIZentren ein Routineeingriff. Zur Verbesserung des Sprachverständnisse im Störschall und der optimierten räumlichen Wahrnehmung sollte die regelhafte bilaterale CI-Versorgung durchgeführt werden.

Bei Patienten, die noch einen funktionell nutzbaren Hörrest im Tieftonbereich, jedoch eine an Taubheit grenzende Schwerhörigkeit im mittleren und im Hochtonbereich haben, kann ebenfalls eine hervorragende Hilfe angeboten werden. Hierbei wird eine spezielle (kürzere) Reizelektrode, idealerweise unter intraoperativer CT-Kontrolle intrakochleär bis an den Bereich des Restgehörs geschoben und dort platziert. Der hochfrequente Anteil der Hörinformation wird nun über elektrische Reize vermittelt, während der für das Sprachverständnis - insbesondere in alltagslauter Umgebung - wichtige Tieftonbereich über ein Hörgerät versorgt wird (elektroakustische Stimulation, EAS). Hierdurch ist eine signifikant bessere Versorgung als mit einem Hörgerät allein möglich. Diese mikrochirurgischen Operationen zur Wiederherstellung des Gehörs sind hoch anspruchsvoll, erfordern ein interdisziplinär zusammenarbeitendes Team und sollten in erfahrenen Zentren erfolgen. Die de- mographische Entwicklung der Bevölkerung lässt erwarten, dass eine steigende Zahl von schwerhörigen Erwachsenen diese Art der kombinierten Versorgung (EAS) benötigt.

Die Ergebnisse eines sehr guten bis hervorragenden Sprachverständnisses sind nur über umfangreiche und zeitintensive Rehabilitationsmaßnahmen möglich. Diese erfordern hoch spezialisiertes Personal, das interdisziplinär HNO-ärztlich, logopädisch, pädagogisch, psychologisch und audiologisch in der Nachsorge eng zusammenarbeitet und in unmittelbarem Austausch mit den operativen Zentren steht oder ihnen angegliedert ist.

Bei Funktionsverlust beider Hörnerven, wie sie beispielsweise bei Neurofibromatose Typ II mit bilateralen Vestibularisschwannomen auftritt, besteht die Möglichkeit der Implantation zentral-auditorischer Systeme. Diese können wieder eine Hörfunktion herstellen, die zwar nicht auf dem hohen Niveau der CI-Systeme liegt, es jedoch ermöglicht, Umweltgeräusche besser wahrzunehmen und das Lippenablesen verbessert.

Diese erweiterten Indikationsspektren stellen den behandelnden Arzt vor neue Aufgaben. Es muss u. a. der richtige Zeitpunkt und die korrekte Modalität (konventionelles CI vs. EAS) für die Implantation gefunden werden. Die CI-Versorgung wird zunehmend komplexer, obwohl man sie heute als Standardeingriff an HNO-Zentren bezeichnen kann. Es ist die Aufgabe, aber auch die Chance des behandelnden HNO-Arztes, die geeigneten Patienten zu selektieren, zu beraten und einer entsprechenden Therapie zuzuführen. 
Aufgrund der Vielschichtigkeit dieses Verfahrens haben wir dem Leitthema „Kochleaimplantat“ 2 Ausgaben mit zahlreichen, aktuellen Übersichten und Originalien gewidmet. Wir wünschen Ihnen bei der Lektüre dieser beiden Themenhefte viel Freude.

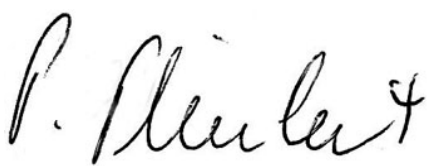

Prof. Dr. Dr. h.c. Peter K. Plinkert (Schriftleitung)
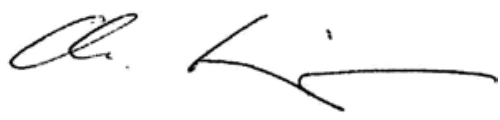

Priv.-Doz. Dr. C. Klingmann (Assistenz-Schriftleitung)

\section{Korrespondenzadressen}

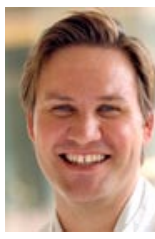

PD Dr. C. Klingmann

Universitäts-Hals-Nasen-

Ohren-Klinik Heidelberg

Im Neuenheimer Feld

400, 69120 Heidelberg

christoph.klingmann@

med.uni-heidelberg.de

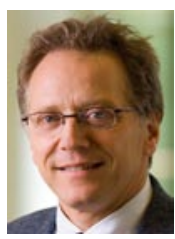

Prof. Dr. P. K. Plinkert

Universitäts-Hals-NasenOhren-Klinik Heidelberg Im Neuenheimer Feld 400, 69120 Heidelberg peter.plinkert@med. uni-heidelberg.de

\section{HNO OP Update 2009}

1. HNO-Operations-Update „Nase \& Nasennebenhöhlen“ - gelungener Auftakt

Das neue Update-Format zu OP-Techniken der Hals-Nasen-Ohren-Heilkunde begeisterte über 120 Teilnehmer bei der Auftaktveranstaltung am 05. und 06. Juni 2009 in Frankfurt am Main.

Das Interesse an diesem innovativen Fortbildungskonzept war vor allem auch bei den HNO-Update-Teilnehmern aus 2008 sehr groß, die das HNO OP Update als weiterführende und praxisnahe Ergänzung zum bereits etablierten HNO Update in Mainz empfunden haben.

Unter der wissenschaftlichen Leitung von Prof. Heinrich Iro, Erlangen, und Prof. Jochen A. Werner, Marburg, wurde den Ärzten und Assistenzärzten aus HNO-Kliniken und HNO-Praxen aus Deutschland, Italien und der Schweiz der neueste Standard bezüglich OP-Techniken, neuen chirurgischen Geräten und Instrumenten sowie neuen diagnostischen Möglichkeiten vermittelt. In lehrreichen Videos erklärte das namhafte Referententeam einzelne OP-Schritte und gab zahlreiche Tipps und Hinweise bezüglich der Umsetzung dieser Methoden und chirurgischen Techniken. Durch 12 verschiedene Vortragsthemen war der Inhalt des HNO OP Updates „Nase \& Nasennebenhöhlen" sehr breit gefächert und deckte von der Anatomie der Nase, Rhinoplastik, Siebbein, Stirnhöhle, Kieferhöhle, Tränenwege bis hin zur Nachsorge und zu Komplikationen ein weites Themenspektrum ab.

Ein Drittel der Vortragszeit wurde jeweils für angeregte Diskussionen zwischen Teilnehmern und Referenten genutzt. Diese Diskussionsrunden sind wichtiger Bestandteil des etablierten Update-Konzepts und wurden von den erfahrenen Moderatoren Prof. Malte Wigand, Erlangen, und Prof. Heinrich Rudert, Hannover, geleitet.

Als besonderes Plus erhielt jeder Teilnehmer das HNO-OP-Manual, das alle Manuskripte der Referenten beinhaltet und als Nachschlagewerk der vorgestellten Techniken dient. Eine CD-ROM mit den Vortragspräsentationen wird den HNO-OP-UpdateTeilnehmern demnächst zugesandt.
Partner des HNO OP Updates ist die Firma KARL STORZ GmbH \& Co. KG, die keinen Einfluss auf die inhaltliche Gestaltung des Seminars nimmt und ohne die diese hochwertige Veranstaltung in diesem Rahmen nicht möglich gewesen wäre.

Aufgrund der hervorragenden Resonanz ist eine weitere Veranstaltung für das nächste Jahr mit einem anderen vertiefenden Themengebiet geplant. Sobald es weitere Informationen hinsichtlich einer Folgeveranstaltung gibt, werden diese auf der Homepage www.hno-op-update.com veröffentlicht.

Bereits im Herbst findet am 27. und 28. November 2009 in der Rheingoldhalle in Mainz das 3. HNO-Update-Seminar statt, das in bewährter Form klinische Studien des vergangenen Jahres aus 14 Themengebieten der Hals-Nasen-Ohren-Heilkunde selektiert, referiert und kommentiert.

Weitere Informationen zu dieser Veranstaltung und Anmeldemöglichkeit auf www.hno-update.com.

Kontakt:

med update $\mathbf{G m b H}$

Sabine Heck

Hagenauer Straße 53

65203 Wiesbaden

Tel.: 0611/73658-17

Fax: 0611/73658-10

E-Mail: sabine.heck@med-update.com 